\title{
Wormlike Micelles in Mixed Amino Acid Surfactant/ Nonionic Surfactant Aqueous Systems and the Effect of Added Electrolytes
}

\author{
Rekha Goswami Shrestha ${ }^{1}$, Carlos Rodriguez-Abreu² and Kenji Aramaki ${ }^{1 *}$ \\ ${ }^{1}$ Graduate School of Environment and Information Sciences, Yokohama National University (79-7 Tokiwadai, Hodogaya-ku, Yokohama 240- \\ 8501, JAPAN) \\ ${ }^{2}$ Institut de Química Avançat de Catalunya. Consejo Superior de Investigaciones Científicas (IQAC/CSIC) (Jordi Girona, 18-26, 08034 \\ Barcelona, SPAIN)
}

\begin{abstract}
The formation of viscoelastic wormlike micelles in mixed amino acid surfactant/nonionic surfactant aqueous systems in the presence of different counterions and salts is reported, and the effects of the different electrolytes on the rheological behavior are discussed. $\mathbf{N}$-dodecanoylglutamic acid (LAD) is neutralized with biologically relevant L-lysine and L-arginine to obtain anionic surfactants (LAD-Lys2, LAD-Arg2) which form aqueous micellar solutions at $25^{\circ} \mathrm{C}$. Addition of a nonionic surfactant, triethyleneglycol mono n-tetradecyl ether $\left(\mathrm{C}_{14} \mathrm{EO}_{3}\right)$, to the aqueous solutions of both LAD-Lys2 and LADArg2 causes the zero-shear viscosity $\left(\eta_{0}\right)$ to increase with $\mathrm{C}_{14} \mathrm{EO}_{3}$ concentration gradually at first, and then sharply, indicating one-dimensional growth of the aggregates and eventual formation of entangled wormlike micelles. Further addition of $\mathrm{C}_{14} \mathrm{EO}_{3}$ ultimately leads to phase separation of liquid crystals. Such a phase separation, which limits the maximum attainable viscosity, takes place at lower $\mathrm{C}_{14} \mathrm{EO}_{3}$ concentrations for LAD-Lys2 compared to LAD-Arg2 systems.

It was found that the rheological behavior of micellar solutions is significantly affected by the addition of $\mathrm{Na}^{+} \mathrm{X}^{-}$salts $\left(\mathrm{X}=\mathrm{Cl}^{-}, \mathrm{Br}^{-}, \mathrm{I}^{-}, \mathrm{NO}_{3}^{-}\right)$. The maximum viscosities obtained for the systems with added salt are all higher than that of the salt-free system, and the onset of wormlike micelle formation shift towards lower nonionic surfactant concentrations upon addition of electrolyte. The maximum attainable thickening effect of anions increases in the order $\mathrm{NO}_{3}^{-}>\mathrm{I}^{-}>\mathrm{Br}^{-}>\mathrm{Cl}^{-}$.

The effect of temperature was also investigated. Phase separation takes place at certain temperature, which depends on the type of anion in the added salt, and decreases in the order $\mathrm{I}^{-}>\mathrm{NO}_{3}^{-}>\mathrm{Br}^{-} \approx \mathrm{Cl}^{-}$, in agreement with Hofmeister's series in terms of amphiphile solubility. The thermoresponsive rheological behavior was also found to be highly dependent on the type of anion, and anomalous trends, i.e. viscosity increase with temperature, were observed for all anions except $\mathrm{Br}^{-}$.
\end{abstract}

Key words: amino acid surfactant, phase behavior, wormlike micelles, rheology, viscoelastic solutions

\section{INTRODUCTION}

Amphiphilic molecules can self-assemble into long cylindrical micelles in aqueous solutions, commonly known as wormlike micelles, which eventually entangle to form viscoelastic networks ${ }^{1-3}$. The equilibrium and dynamics of these structures can be affected by changes in temperature, dilution, variation of salt concentration, or the addition of oils or polymers ${ }^{4,5)}$. In the case of charged micelles (ionic surfactant systems), micellar growth occurs as a consequence of reduction of surfactant head group's interaction, which is in general induced by salt ions, strongly binding counterions or sometimes by cosurfactants ${ }^{1,2,6-11}$. The molecules packed at the hemispherical ends of long aggregates have an excess free energy (so-called "end-cap energy") in comparison to the molecules at the cylindrical part; therefore, the "end-cap energy" acts as a driving force for the linear growth of the cylindrical micelles. The system can minimize the free energy by reducing the num-

\footnotetext{
* Correspondence to: Kenji Aramaki, Graduate School of Environment and Information Sciences, Yokohama National University, 79-7 Tokiwadai, Hodogaya-ku, Yokohama 240-8501, JAPAN

E-mail: aramakik@ynu.ac.jp
}

Accepted January 15, 2009 (recieved for review December 11, 2008)

Journal of Oleo Science ISSN 1345-8957 print / ISSN 1347-3352 online

http://www.jstage.jst.go.jp/browse/jos/ 


\section{R.G. Shrestha, C. Rodriguez-Abreu and K. Aramaki}

ber of free end-caps. Hence, wormlike aggregates are favored over short rod-like micelles. Above a certain concentration, these wormlike micelles entangle with each other and form a transient network responsible for its viscoelastic properties.

Wormlike micelles cover a wide range of applications. They are mostly used in food, emulsions, cosmetics, paints, pharmaceuticals, adhesives, and many household products. Therefore, knowledge on the structure and dynamics of wormlike micellar systems is important for the optimization of these applications ${ }^{12-16)}$. Salt induced wormlike micelles in ionic surfactants have been widely reported ${ }^{17-20}$. A few papers are available in the literature on the rheological behavior of wormlike micelles in mixed system of ionic/nonionic surfactant systems ${ }^{21-25}$. Cationic surfactant in the presence of strongly binding counterions or excess salts, forms viscoelastic wormlike micelles ${ }^{26,27}$. Similarly, the formation of viscoelastic wormlike micelles in mixed systems of cationic/anionic surfactants is well studied ${ }^{10,28}$. However, anionic surfactants are used in a greater volume than any other surfactants, because of their highly potent detergency and low cost of manufacture. Amino acid based anionic surfactants (both natural and synthetic types) are one of the environmentally friendly anionic surfactants having many applications in cosmetic and toiletry products due to their mildness to the skin. Recently, we have found that the N-dodecanoylglutamic acid, designated as LAD (an amino acid based anionic surfactant) upon neutralization with triethanolamine (TEA) or L-lysine forms viscoelastic wormlike micelles in mixed systems of $\mathrm{LAD} /$ cationic surfactant or $\mathrm{LAD} /$ nonionic surfactant without addition of any salts ${ }^{29,30)}$. However, there is no report on this kind of surfactants concerning the effects of salts (often present in formulations) or the effect of changing organic counterions, on the phase behavior and rheological properties of amino acid surfactant aqueous systems.

In this paper we report on the phase behavior and formation of wormlike micelles in mixed LAD-X/nonionic surfactant systems where $\mathrm{X}$ are biologically relevant organic counterions (i.e., L-lysine and L-arginine), and the effect of different salts on the rheological behavior.

\section{EXPERIMENTAL}

\subsection{Materials}

$\mathrm{N}$-dodecanoylglutamic acid (designated as LAD) and Larginine were obtained from Ajinomoto Co., Inc. Japan. Homogeneous tri-ethyleneglycol mono n-tetradecyl ether $\left(\mathrm{C}_{14} \mathrm{EO}_{3}\right)$ was purchased from Nikko Chemicals Co., Ltd. Japan. Sodium Chloride, Sodium Bromide, Sodium Iodide and Sodium Nitrate were purchased from Wako Pure Chemicals Industries, Ltd. Japan. The purity of all chemi- cals was higher than $99 \%$, so they were used as received without further purification. The molecular structure of LAD is presented in Scheme 1.

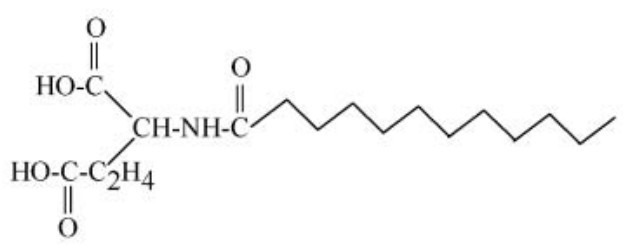

Scheme 1 Molecular Structure of N-Dodecanoylglutamic Acid (LAD).

\subsection{Methods}

\subsubsection{Phase behavior}

First of all, aqueous binary phase behavior of neutralized LAD was studied at $25^{\circ} \mathrm{C}$. For this purpose, different samples $(\sim 0.50 \mathrm{~g}$ each) of composition range $2-50 \mathrm{wt} \%$ were prepared in clean and dry glass ampoules (size $12 \mathrm{~mm} \times$ $100 \mathrm{~mm}$ ) and immediately flame sealed. The chemicals were properly weighed and the total uncertainty for the values of concentration of each component is $\pm 0.01 \%$. The samples were mixed with vortex mixer, and repeated centrifugation in order to get homogeneity. After mixing, the samples were put into a water bath with temperature set to $25^{\circ} \mathrm{C}$ with the accuracy of the thermometer $\pm 0.5^{\circ} \mathrm{C}$. The samples were left for $24 \mathrm{~h}$ before identifying the equilibrium phases. Next, partial ternary phase diagrams were constructed at $25^{\circ} \mathrm{C}$. Samples with compositions ranging from 5 to $40 \mathrm{wt} \%$ surfactant $(1 \mathrm{~g}$ each) were prepared in clean and dry glass ampoules with screw cap. Samples were properly mixed with vortex mixer, and repeated centrifugation. Finally, ternary phase diagrams were constructed by adding $\mathrm{C}_{14} \mathrm{EO}_{3}$ and following the method of titration. In the titration method, first $\sim 0.2 \mathrm{wt} \% \mathrm{C}_{14} \mathrm{EO}_{3}$ was added into the micellar solution of neutralized LAD, and then mixed properly, and finally kept into a water bath at $25^{\circ} \mathrm{C}$ for an hour prior to identify the equilibrium phase. The addition of $\mathrm{C}_{14} \mathrm{EO}_{3}$, mixing, phase identification processes were continued until phase separation to liquid crystal takes place. In order to confirm the phase boundary, sealed ampoules containing a required amount of reagents were homogenized and kept in a water bath at $25^{\circ} \mathrm{C}$ for $24 \mathrm{~h}$ for equilibration. Equilibrium phases were identified by visual observations (through crossed polarizer). Millipore water was used in preparing all the solutions.

2.2.2 Rheological measurements

All the samples ( $3 \mathrm{~g}$ each) for rheological measurements were prepared taking required amount of chemicals in clean and dry glass bottles $(20 \mathrm{~mL})$ with screw cap. The chemicals were properly weighed so that the total uncertainty for the values of concentration of each component is $\pm 0.01 \%$. Samples were homogenized and kept in a thermostated bath at $25^{\circ} \mathrm{C}$ with the accuracy of the thermome- 
ter $\pm 0.5^{\circ} \mathrm{C}$ for at least $24 \mathrm{~h}$ before the measurements. Viscous samples were mixed using a magnetic stirrer. The rheological measurements were performed on an AR-G2 stress-controlled rheometer (TA Instruments) equipped with a Peltier-based temperature controller using coneplate geometries (cone diameter: $40 \mathrm{~mm}$; cone angle: $1^{\circ}$ ). A sample cover provided with the instrument was used to minimize the change in sample composition due to evaporation during the measurement. Frequency sweep measurements were performed in the linear viscoelastic regime of the samples, as determined previously by dynamic strain sweep measurements.

\section{RESULTS AND DISCUSSION}

\subsection{LAD/non-ionic surfactant/water systems}

\subsubsection{Phase behavior}

LAD possesses a relatively high $\mathrm{Krafft}$ point $\left(58^{\circ} \mathrm{C}\right)$. However, when both the carboxylic acid groups of the LAD are neutralized with TEA or lysine, the Krafft point is reduced below room temperature, and a micellar solution is formed at $25^{\circ} \mathrm{C}^{29,30)}$. In the present study, we have used arginine as a neutralizing agent (counter ion) to compare its phase and rheological behavior with that of the LAD-lysine system.

Figure 1 shows the effect of neutralization degree of LAD on the Krafft temperature for L-arginine and L-lysine. The Krafft point of LAD-arginine systems keeps around $50^{\circ} \mathrm{C}$ up to a neutralization ratio of $\mathrm{Arg} / \mathrm{LAD}=1$; above this value, the Krafft point decreases sharply and for $\mathrm{Arg} / \mathrm{LAD}=2$, isotropic micellar solution is found above $0^{\circ} \mathrm{C}$. On the other hand, when the counterion is L-lysine (Figure 1b), the Krafft point decreases almost monotonically with the neutralization degree up to Lys $/ \mathrm{LAD}=1.5$; above this value, the Krafft points remain constant around $0^{\circ} \mathrm{C}$. Note that there are regions in which a solid phase and an isotropic solution coexist; such regions are narrower for LAD-lysine system in comparison to the LAD-arginine system. The neutralization ratios to obtain isotropic solutions at room temperature are $\mathrm{Arg} / \mathrm{LAD}=2$ for LAD-arginine system and Lys $/ \mathrm{LAD}=0.8$ for LAD-lysine system. In the following experiments we have used LAD-arginine and LAD-lysine surfactants with a neutralization degree equal to 2 , abbreviated as LAD-Arg2 and LAD-Lys2, respectively.

To explore the effects of mixing with nonionic surfactants, we constructed partial ternary phase diagrams of LAD-Arg2/water $/ \mathrm{C}_{14} \mathrm{EO}_{3}$ and LAD-Lys2/water $/ \mathrm{C}_{14} \mathrm{EO}_{3}$ system in the dilute regions at $25^{\circ} \mathrm{C}$ as shown in Fig. 2. In the LAD-Arg2/water binary system, isotropic solutions are formed over a wide range of surfactant concentrations. The viscosity of dilute aqueous solution of LAD-Arg2 is comparable to pure water, which suggests that micelles are spherical above the critical micelle concentration (CMC).

For the binary LAD-Arg2 aqueous system (LAD-Arg2water axis), the liquid crystal region just adjacent to the $\mathrm{L}_{1}$ region is a direct micellar cubic phase, as confirmed by Small Angle X-ray (SAXS) measurements (see Fig. 2c). Such a cubic phase has been found in other binary amino acid surfactant-water systems as well ${ }^{311}$. On the other hand, $\mathrm{C}_{14} \mathrm{EO}_{3}$ is insoluble in water and therefore two liquid phases coexist in the $\mathrm{C}_{14} \mathrm{EO}_{3}$ - water axis.

Upon successive addition of $\mathrm{C}_{14} \mathrm{EO}_{3}$ to dilute aqueous solution of LAD-Arg2, the viscosity increases gradually at first, and then sharply. Further addition of $\mathrm{C}_{14} \mathrm{EO}_{3}$ results in phase separation of birefringent liquid crystals.

The binary system LAD-Lys2/water (LAD-Lys2-water axis) also forms an isotropic solution at low concentrations

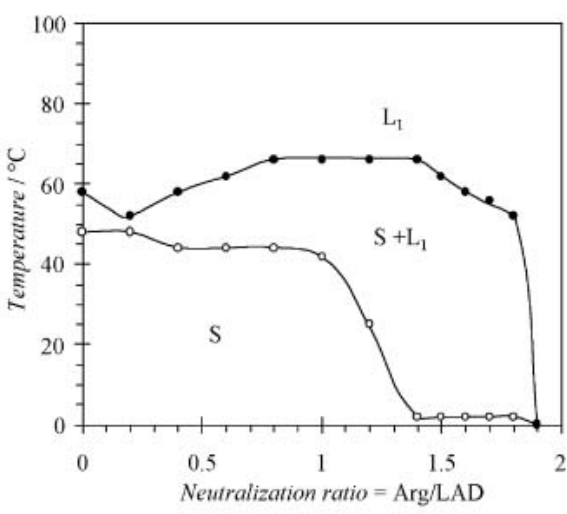

(a)

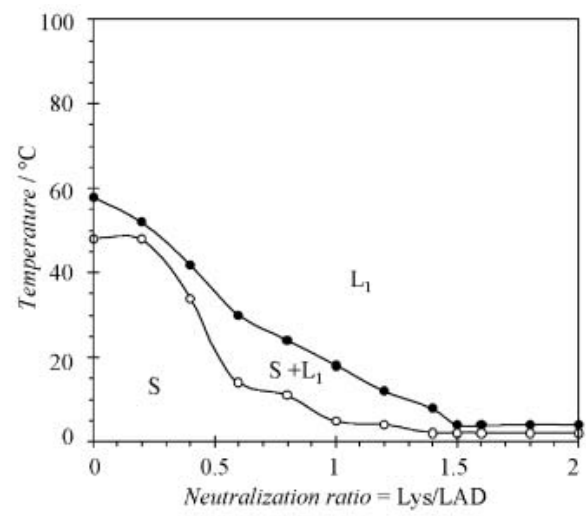

(b)

Fig. 1 Effect of Neutralization Degree of LAD on the Phase Behavior of (a) LAD-Arginine and (b) LAD-Lysine Aqueous Systems.

$\mathrm{S}$ is a solid phase, and $L_{1}$ is a micellar solution, respectively. The $\mathrm{LAD}$ concentration is fixed at $10 \mathrm{wt} \%$. 


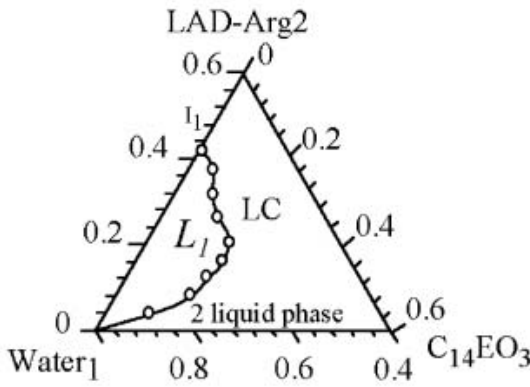

(a)

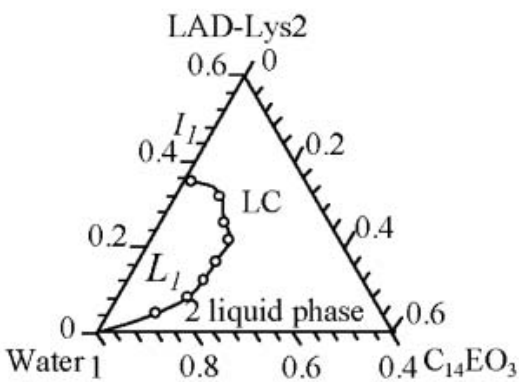

(b)

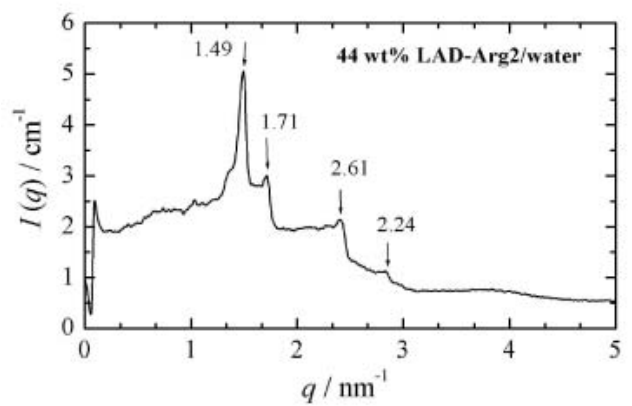

(c)

Fig. 2 Partial Ternary Phase Diagrams of (a) LAD-Arg2/water/ $\mathrm{C}_{14} \mathrm{EO}_{3}$ and (b) LAD-Lys $2 /$ Water $/ \mathrm{C}_{14} \mathrm{EO}_{3}$ Systems at $25^{\circ} \mathrm{C}$ where $L_{1}$ is Micellar Solution with High Fluidity (possibly spherical micelles).

LC represents a liquid crystal region. $I_{1}$ is discrete cubic phase (Figure $b$ is adapted from ref. 30). (c) SAXS pattern of discrete cubic phase in LAD-Arg2/water binary system at $25^{\circ} \mathrm{C}$.

but transforms into a cubic phase at lower surfactant concentrations as compared to LAD-Arg2. The other features of the LAD-Lys2/water $/ \mathrm{C}_{14} \mathrm{EO}_{3}$ phase diagram are similar to that of LAD-Lys $2 /$ water $/ \mathrm{C}_{14} \mathrm{EO}_{3}$ system, namely, highly viscous solutions were also found in the vicinity of the $\mathrm{LC}$ region.

\subsubsection{Rheological behavior}

In Fig. 3, we present the steady shear-rate $(\dot{\gamma})-$ viscosity $(\eta)$ curves for $10 \mathrm{wt} \% \mathrm{LAD}-\mathrm{Arg} 2+\mathrm{C}_{14} \mathrm{EO}_{3}$ system at different mixing fraction of $\mathrm{C}_{14} \mathrm{EO}_{3}$, expressed in wt\% of $\mathrm{C}_{14} \mathrm{EO}_{3}$ in total system at $25^{\circ} \mathrm{C}$. At lower $\mathrm{C}_{14} \mathrm{EO}_{3}$ concentrations, $\eta$ is small, close to that of pure solvent (water) and independent of $\dot{\gamma}$, i.e., Newtonian flow behavior is observed. However, as concentration increases, $\eta$ increases and shear thinning is observed, which is a typical behavior of wormlike micelles. As can be seen from Fig. 3(a), the flow behavior is Newtonian over wide range of shear rate at $14.5 \mathrm{wt} \% \mathrm{C}_{14} \mathrm{EO}_{3}$, but shear thinning occurs at $\dot{\gamma} \geq 100 \mathrm{~s}^{-1}$. With further increasing concentration, the critical shearrate $\dot{\gamma}_{c}$ (the shear-rate at which shear thinning occurs) shifts gradually to the lower values, and the viscosity in the plateau region (low $\dot{\gamma}$ region) increases. This phe- nomenon highlights the fact that the system is getting more structured. The observed steady-shear rheological behavior is a typical of network structure formed by wormlike micelles. The network structure is deformed by applying a shear, and hence, shear thinning occurs due to alignment of aggregates under flow if the deformation is faster than the time required for regaining equilibrium.

The effect of adding $\mathrm{C}_{14} \mathrm{EO}_{3}$ to LAD-Arg2/water and LAD-Lys2/water systems on zero-shear viscosity $\left(\eta_{0}\right)$ is also shown in Fig. 3(b). An almost monotonic increase in $\eta_{0}$ with $\mathrm{C}_{14} \mathrm{EO}_{3}$ concentration can be seen in both systems; further addition of $\mathrm{C}_{14} \mathrm{EO}_{3}$ leads to phase separation, which occurs at lower nonionic surfactant concentrations for LAD-Lys2 than for LAD-Arg2 system, as already indicated by the phase diagrams in Fig. 2. Therefore, the maximum attainable viscosity is higher for LAD-Arg2 at the given composition.

\subsection{LAD-Arg2/non-ionic surfactant/water systems with added salt}

3.2.1 Rheological behavior

Due to the usual presence of electrolytes in formulations, 


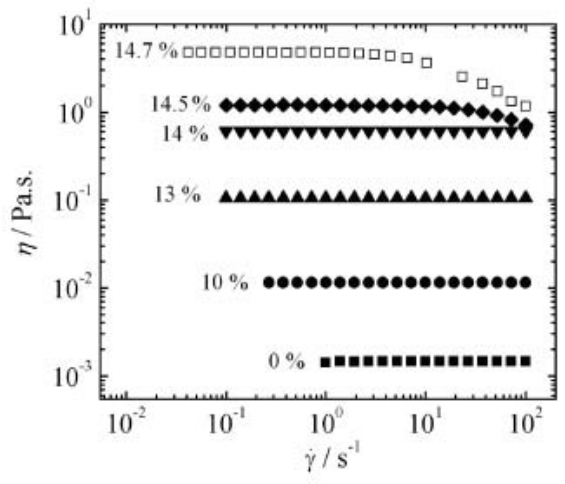

(a)

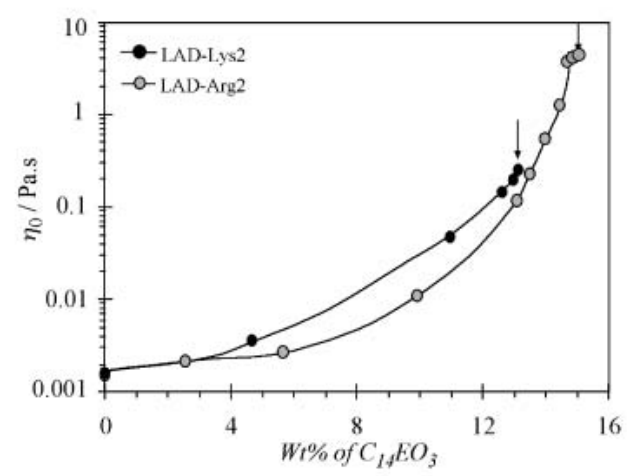

(b)

Fig. 3 (a) Viscosity vs. Shear Rate of the LAD-Arg2/Water $/ \mathrm{C}_{14} \mathrm{EO}_{3}$ System at Different Mixing Fractions of $\mathrm{C}_{14} \mathrm{EO}_{3}$ (expressed as wt $\%$ besides each set of data). (b) Zeroshear Viscosity vs. $w \mathrm{t} \%$ of $\mathrm{C}_{14} \mathrm{EO}_{3}$ for LAD-Arg2/Water/ $\mathrm{C}_{14} \mathrm{EO}_{3}$ and LAD-Lys $2 /$ Water/ $\mathrm{C}_{14} \mathrm{EO}_{3}$ Systems at $25^{\circ} \mathrm{C}$.

The LAD-Arg2 and LAD-Lys2 concentrations in water are kept at $10 \mathrm{wt} \%$. Arrows indicate the $\mathrm{C}_{14} \mathrm{EO}_{3}$ concentration at which phase separation takes place.

the effect of a series of salts with a common cation $(\mathrm{Na})$ on the rheological behavior of LAD- $\mathrm{Arg} 2 / \mathrm{C}_{14} \mathrm{EO}_{3}$ aqueous systems was studied. As can be seen in Fig. 4, salts modify the rheological behavior significantly. Namely, $\eta_{0}$ curves become steeper and shift towards lower $\mathrm{C}_{14} \mathrm{EO}_{3}$ concentration compared to the salt-free system, indicating that a lower amount of $\mathrm{C}_{14} \mathrm{EO}_{3}$ is required for micellar growth when salt is added. In addition, the amount of $\mathrm{C}_{14} \mathrm{EO}_{3}$ needed for the onset of thickening for the different salts seems to increase in the order $\mathrm{I}^{-}>\mathrm{NO}_{3}{ }^{-}>\mathrm{Br}^{-}>\mathrm{Cl}^{-}$, which is in agreement with the Hofmeister series in terms of amphiphile solubility. Hence, the salting-out effect is playing a role in the onset of micellar growth. For $\mathrm{NaCl}$ and NaI slight viscosity maxima are observed, whereas for $\mathrm{NaNO}_{3}$ and $\mathrm{NaBr}$, viscosity increases continuously until phase separation takes place. The maximum thickening effect of anions, i.e. the maximum attainable viscosity, increases in the order $\mathrm{NO}_{3}{ }^{-}>\mathrm{I}^{-}>\mathrm{Br}^{-}>\mathrm{Cl}^{-}$.

The effects of salt concentration on the steady-shear rheology, and hence, on $\eta_{0}$ for a particular salt $\left(\mathrm{NaNO}_{3}\right)$ is shown in Fig. 5. For the sake of comparison, data of the salt free system is also included in the figure. The $\eta_{0}$ curves shift towards lower $\mathrm{C}_{14} \mathrm{EO}_{3}$ concentration with increasing salt concentration indicating that the salt favors micellar growth. In order to figure out the net salt concentration effects on $\eta_{0}$, we have plotted $\eta_{0}$ as a function of salt concentration at fixed concentrations of LADArg2 and $\mathrm{C}_{14} \mathrm{EO}_{3}$ (see Fig. 5b). It can be seen that at fixed LAD-Arg2 $/ \mathrm{C}_{14} \mathrm{EO}_{3}$ mixing ratios, there is an inflexion point at a certain $\mathrm{NaNO}_{3}$ concentration, which can be assigned to the onset of micellar growth. Curves shift to higher viscosities with increasing $\mathrm{C}_{14} \mathrm{EO}_{3}$ concentration. It is gener-

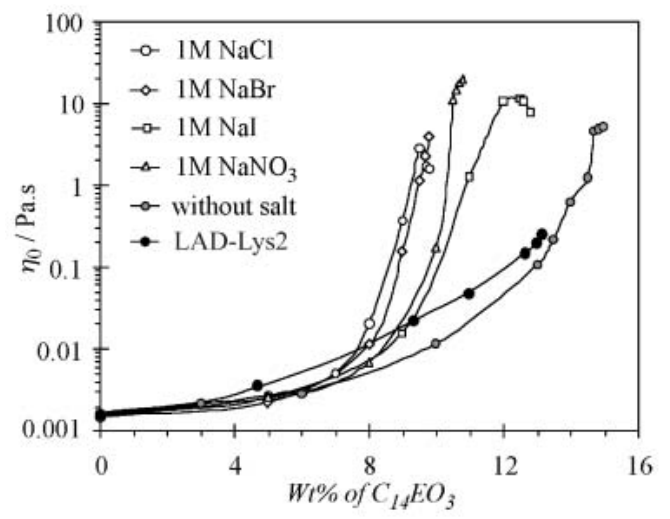

Fig. 4 Zero-shear Viscosity vs. $\mathrm{C}_{14} \mathrm{EO}_{3}$ Concentration for Different Salts in LAD-Arg2/ $\mathrm{C}_{14} \mathrm{EO}_{3}$ Aqueous Systems.

The concentration of LAD-Arg2 is fixed at $10 \mathrm{wt} \%$, and salt concentration is fixed at $1 \mathrm{M}$. All the measurements were carried out at $25^{\circ} \mathrm{C}$.

ally admitted that increasing the salt concentration amounts to an increase in the curvature energy of surfactant molecules in the end-cap region relative to the one in the cylindrical body of the micelle. This leads to an increase in micellar length.

As can be seen in Fig. 5c, the maximum attainable zeroshear viscosity, $\eta_{0 \max }$ (indicated by small arrows in Fig. 5a) first increases with $\mathrm{NaNO}_{3}$ concentration up to $0.3 \mathrm{M}$, and then decreases. It is possible that the salt first favors micellar growth, but after a certain concentration, micellar shortening or branching occurs and maximum zero-shear 


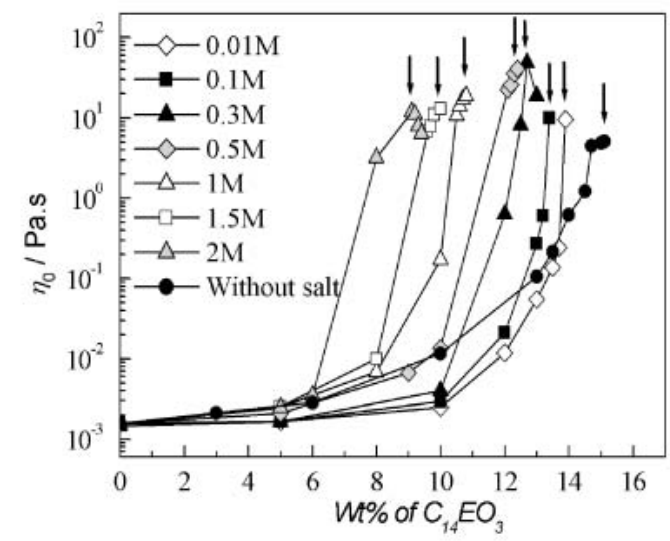

(a)

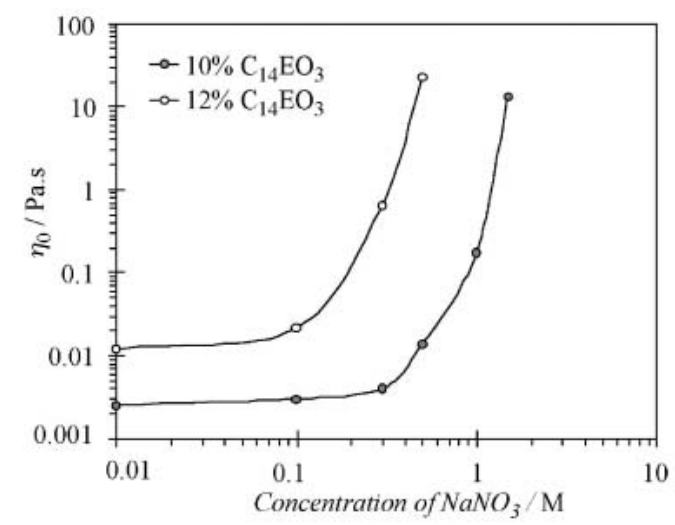

(b)

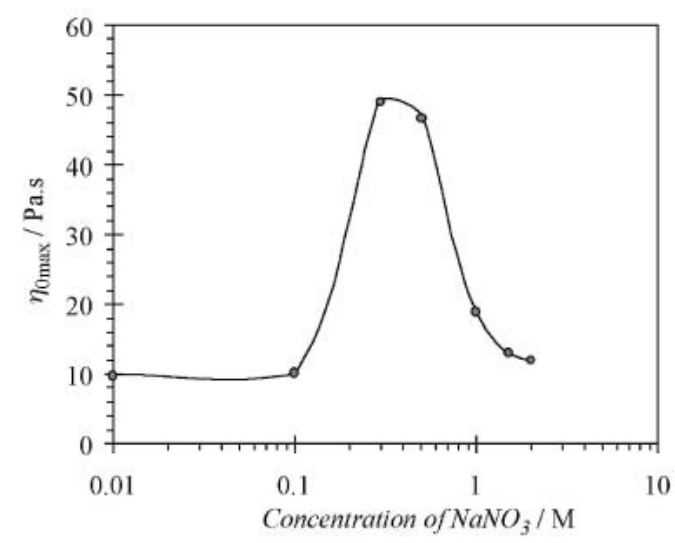

(c)

Fig. 5 (a) Zero-shear Viscosity vs. $\mathrm{C}_{14} \mathrm{EO}_{3}$ Concentration at Different Concentrations of $\mathrm{NaNO}_{3}$.

The concentration of LAD-Arg2 is fixed at $10 \mathrm{wt} \%$, (b) effects of salt concentration on $\eta_{0}$ at fixed LADArg2 concentration (10 wt $\%$ ) for two different $\mathrm{C}_{14} \mathrm{EO}_{3}$ concentrations, and (c) $\eta_{0 \max }$ (indicated by small arrows in panel (a)) as a function of $\mathrm{NaNO}_{3}$ concentration. All the measurements were carried out at $25^{\circ} \mathrm{C}$. viscosity decreases. $\eta_{0 \max }$ is also related to the $\mathrm{C}_{14} \mathrm{EO}_{3}$ concentration for phase separation, which decreases with $\mathrm{NaNO}_{3}$ concentration.

Viscoelastic properties of the wormlike micelles as a function of nonionic surfactant concentrations were investigated by oscillatory-shear (frequency sweep) measurements. Generally, dynamics of wormlike micelles under oscillatory-shear is described by considering two different processes. When a small strain is applied, the stress relaxation occurs by reptation, that is, a reptile-like motion of the micelle along its own contour, with an associated relaxation time, $\tau_{\text {rep }} \sim(\bar{L})^{3}$, where $\bar{L}$ is the micellar contour length ${ }^{3)}$. Beside reptation, micelles may also undergo reversible scission, with a characteristic time, $\tau_{b} \sim(\bar{L})^{-132)}$. When the time scale of the scission for an average micellar contour length is too short in comparison to the time scale of the reptation $\left(\tau_{\text {rep }} \gg \tau_{b}\right)$, the viscoelastic micellar solutions behave as a Maxwell fluid ${ }^{33,34)}$ with a single relaxation time $^{3)}$, given by

$$
\tau_{R}=\left(\tau_{b} \tau_{\text {rep }}\right)^{\frac{1}{2}} \sim \bar{L}
$$

The variation of the elastic or storage modulus $G^{\prime}(\omega)$ and the viscous or loss modulus $G^{\prime \prime}(\omega)$ as a function of oscillatory-shear frequency, $\omega$, is described by the following relations:

$$
\begin{aligned}
& G^{\prime}(\omega)=\frac{\omega^{2} \tau_{R}^{2}}{1+\omega^{2} \tau_{R}^{2}} G_{0} \\
& G^{\prime \prime}(\omega)=\frac{\omega \tau_{R}}{1+\omega^{2} \tau_{R}^{2}} G_{0}
\end{aligned}
$$

where $G_{0}$ is the plateau modulus. At high frequencies, $G^{\prime}$ tends to attain a constant value equal to $G_{0}$. The relaxation time $\tau_{R}$ may be estimated from the $G^{\prime}-G^{\prime \prime}$ crossover frequency, that is $\tau_{R}=1 / \omega$, when $G^{\prime}=G^{\prime \prime}$.

Figure 6a shows a plot of the elastic $\left(G^{\prime}\right)$ and loss $\left(G^{\prime \prime}\right)$ moduli as a function of oscillatory frequency $(\omega)$ for different $\mathrm{C}_{14} \mathrm{EO}_{3}$ concentrations in the LAD-Arg2/ $\mathrm{C}_{14} \mathrm{EO}_{3} / 0.3 \mathrm{M}$ $\mathrm{NaNO}_{3}$ system at $25^{\circ} \mathrm{C}$. There is a clear viscoelastic behavior: at low frequencies, the viscous modulus $G^{\prime \prime}$ is larger than the elastic modulus $\left(G^{\prime}\right)$ and the system behaves like a liquid; at high frequencies, $G^{\prime}>G^{\prime \prime}$ and the system behaves like a solid. The system follows the Maxwell model of relaxation in the low frequency range, but departs from it at high frequencies, which might be attributed to the existence of multiple relaxation processes. This deviation is thought to have arisen from a transition of the relaxation mode from "slower" reptation to "faster" relaxation modes, such as the Rouse mode ${ }^{11,35)}$. Maxwellian-type oscillatory rheological behavior of viscous micellar solutions, such as that shown in Fig. 6, can be related to the transient network formed by the entanglement of wormlike micelles ${ }^{11}$.

As evident from the Maxwell equations, at low frequency 


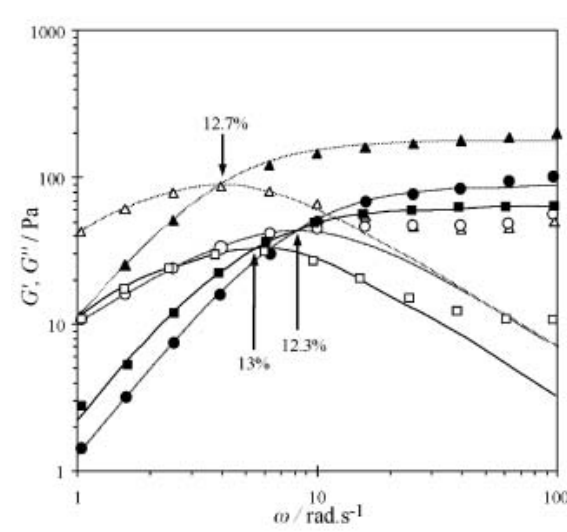

(a)

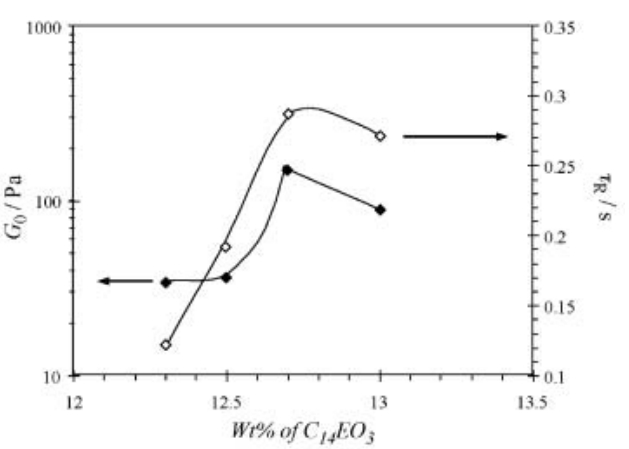

(b)

Fig. 6 (a) Variation of Storage Modulus $\left(G^{\prime}\right)$, and Loss Modulus $\left(G^{\prime \prime}\right)$ as a Function of Oscillatory Frequency $(\omega)$ for Different $\mathrm{C}_{14} \mathrm{EO}_{3}$ Concentrations in the LAD$\operatorname{Arg} 2 / \mathrm{C}_{14} \mathrm{EO}_{3} / 0.3 \mathrm{M} \mathrm{NaNO}_{3}$ System at $25^{\circ} \mathrm{C}$.

The LAD-Arg2 concentration is kept at $10 \mathrm{wt} \%$. The solid lines represent the best fit to the Maxwell model. Arrows indicate the $G^{\prime}-G^{\prime \prime}$ crossover (b) Variation of plateau modulus, $G_{0}$ (closed symbols) and relaxation time, $\tau_{R}$ (open symbols) for the system mentioned in (a).

region, $\omega \ll \omega_{c}$, therefore $G^{\prime}$ and $G^{\prime \prime}$ scale with $\omega$ according to $G^{\prime} \sim \omega^{2}$ and $G^{\prime \prime} \sim \omega$. On the other hand, in the region of $\omega$ $\gg \omega_{c}, G^{\prime}$ attains a plateau value equal to $G_{0}$, whereas $G^{\prime \prime}$ shows a monotonic decrease. The shear frequency corresponding to the $G^{\prime}$ and $G^{\prime \prime}$ crossover, $\omega_{c}$, is equivalent to the inverse of relaxation time, $\tau_{R}$. Considering reptation or diffusion of wormlike micelles along its own contour as the mechanism of stress relaxation in the entangled network, the magnitude of $\tau_{R}$ is related to the average length of the wormlike micelles, whereas $G_{0}$ is related to the number density of entanglement in the transient network. The parameters $G_{0}$ and $\tau_{R}$ are related to $\eta_{0}$ by following relation:

$$
\eta_{0}=G_{0} \tau_{R}
$$

As can be seen from Fig. 6a, with increasing $\mathrm{C}_{14} \mathrm{EO}_{3}$ concentration, the frequency at which the $G^{\prime}$ and $G^{\prime \prime}$ meet shifts towards lower values and attains a minimum at the composition for the viscosity maximum $\left(12.7 \%\right.$ of $\left.\mathrm{C}_{14} \mathrm{EO}_{3}\right)$. With further increase in $\mathrm{C}_{14} \mathrm{EO}_{3}$ concentration, viscosity decreases and finally phase separation occurs. As can be seen from Fig. $6 \mathrm{~b}, G_{0}$ and $\tau_{R}$ increase with $\mathrm{C}_{14} \mathrm{EO}_{3}$ concentration, show a maximum, and then decrease similarly to the case of viscosity as a function of $\mathrm{C}_{14} \mathrm{EO}_{3}$ concentration. The initial increasing trend can be attributed to the onedimensional micellar growth and increased density (i.e., decreased mesh size) in the network structure of wormlike micelles. On the other hand, the decrease could be attributed to morphology changes in the entangled micelles, although the reasons of this behavior are not clear yet; a possibility would be micellar breaking as suggested from Eq. 1.

The rheological parameters derived from the Maxwell model fitting to the experimental data for salt added systems are presented in Fig. 7. The figure shows the variation of $G_{0}$ and $\tau_{R}$ as a function of $\mathrm{C}_{14} \mathrm{EO}_{3}$ concentration at different salt concentrations for a given salt $\left(\mathrm{NaNO}_{3}\right)$. For the sake of comparison $G_{0}$ and $\tau_{R}$ of the salt free system are also included in the figure.

Both $G_{0}$ and $\tau_{R}$ increases with $\mathrm{C}_{14} \mathrm{EO}_{3}$ concentration for all concentrations of the salt. The increase of $G_{0}$ with $\mathrm{C}_{14} \mathrm{EO}_{3}$ concentration indicates that the number of entanglements in the system increases and a more "rigid" structure is formed. Besides, the micellar length increases as anticipated from the increasing trend of $\tau_{R}$. Thus, the viscosity increase with $\mathrm{C}_{14} \mathrm{EO}_{3}$ concentration in all the systems can be attributed to both micellar growth and increased network density of the micellar structure, i.e., number of entanglements.

As mentioned before, the increase of salt concentration in a particular salt system reduces the amount of nonionic surfactant needed to induce the viscosity increase, i.e., viscosity curves shift towards lower $\mathrm{C}_{14} \mathrm{EO}_{3}$ concentrations. Irrespective of the salt concentrations, $G_{0}$, and $\tau_{R}$ follow an increasing trend with $\mathrm{C}_{14} \mathrm{EO}_{3}$ concentration and are higher than the values obtained for salt free system. Thus, it can be concluded that the higher viscosity observed with salt incorporated systems are due to higher entanglement density of wormlike micelles and also due to longer axial 


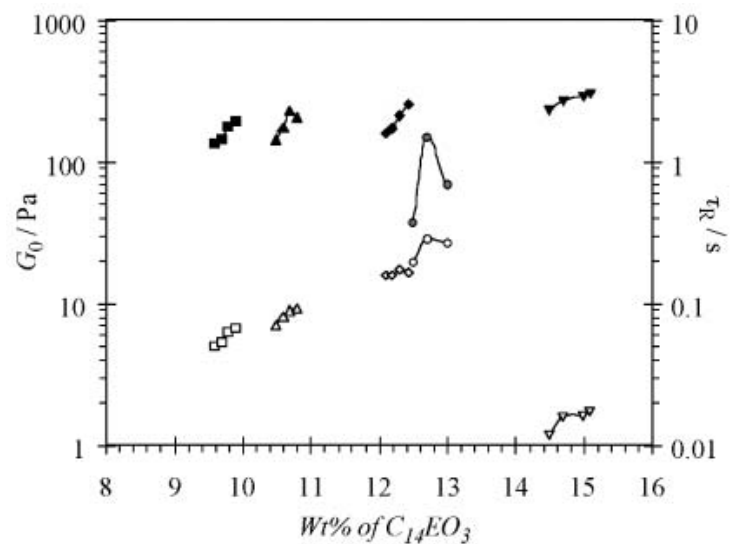

Fig. 7 Variation of Rheological Parameters $G_{0}$ and $\tau_{R}$ as a Function of $\mathrm{C}_{14} \mathrm{EO}_{3}$ Concentration at Different $\mathrm{NaNO}_{3}$ Concentration.

Closed and open symbols represent $G_{0}$ and $\tau_{R}$, respectively. Circle $=0.3 \mathrm{M}$, diamond $=0.5 \mathrm{M}$, triangle $=1 \mathrm{M}$, square $=1.5 \mathrm{M}$, and inverted triangle $=$ without salt. The concentration of LAD-Arg2 is fixed to $10 \mathrm{wt} \%$. All the measurements were carried out at $25^{\circ} \mathrm{C}$.

length of the wormlike micelles. Minute observation of Fig. 7 reveals that although the values of $G_{0}$ are comparable at all salt concentrations studied, $\tau_{R}$ differ, and it has maximum at $0.3 \mathrm{M} \mathrm{NaNO}_{3}$. Note that this is the composition at which the maximum thickening effect (maximum $\eta_{0 \max }$ ) was observed (see Fig. 5c). Thus, the increase of $\eta_{0 \max }$ with salt concentration at the beginning can be attributed to the micellar growth and the entanglement between them. On the other hand, the decrease in the maximum zero-shear viscosity above a certain salt concentration is possibly due to micelle shortening or branching. Addition of salts at the initial stage screens surfactant head group interaction and favors the micellar growth by reducing the interfacial curvature of the aggregate. However, after a certain concentration (say when a saturation of salt at the micellar interface is reached), the interaction among the salt ions becomes crucial, which in turn, could reduce the screening tendency of the salt. Hence, the interfacial curvature tends to increase, which results in shortening of rod-like micelles. However, still there is a possibility of micellar branching.

3.2.2 Thermoresponsive behavior

Figure 8 displays the results on the effect of temperature on rheological behavior of LAD-Arg2/ $\mathrm{C}_{14} \mathrm{EO}_{3}$ aqueous systems in the presence of salts. The experiment was conducted for all the samples up to the temperature at which phase separation takes place. It was found that the phase separation temperature decreases in the order $\mathrm{I}^{-}>\mathrm{NO}_{3}{ }^{-}$ $>\mathrm{Cl}^{-} \approx \mathrm{Br}^{-}$. This is in agreement with Hofmeister's series

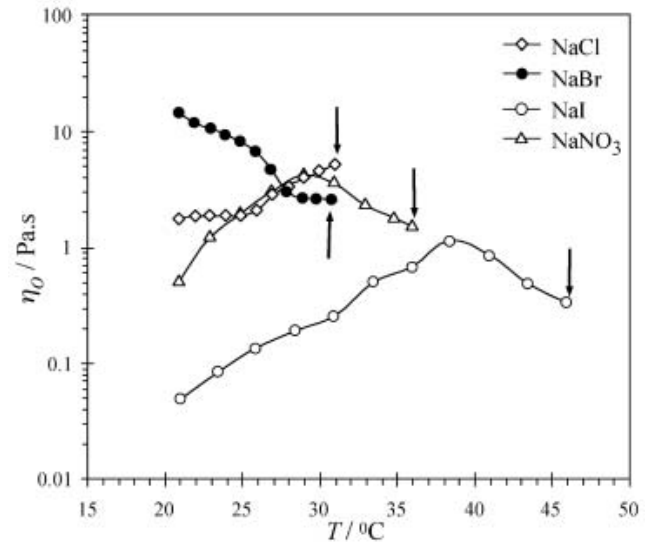

Fig. 8 Variation of $\eta_{0}$ as a Function of Temperature for 10 wt $\%$ LAD-Arg2/water $+9.5 \mathrm{wt}^{0} \% \mathrm{C}_{14} \mathrm{EO}_{3}$ at Fixed Salt Concentration (1 M) for Different Salt Systems. The arrows in the figure indicate the phase separation temperatures for the systems.

in terms of amphiphile solubility; the same trend has been observed for aqueous solutions of thermoresponsive polymers ${ }^{36,37)}$. It is interesting to note that the temperature dependence of viscosity shows different trend depending upon the nature of anion species present in the systems. The viscosity of the system with $\mathrm{NaI}$ and $\mathrm{NaNO}_{3}$ first increases with temperature, reaching a maximum and then decreases. For $\mathrm{NaCl}$ samples the behavior is qualitatively similar at low temperatures; however, no maximum is observed below the phase separation temperature. On the other hand, for $\mathrm{NaBr}$ samples, the viscosity decreases almost continuously with temperature. The exact reason behind the phenomenon is not clear at present.

Oscillatory measurements as a function of temperature for $\mathrm{NaBr}$ and $\mathrm{NaNO}_{3}$ systems are shown in Fig. 9. The values of $G_{0}$ and $\tau_{R}$ calculated by fitting the experimental data to the Maxwell equations are plotted in Fig. 9(a) and (b). In the case of $\mathrm{NaBr}$ system, it is observed that the relaxation time, $\tau_{R}$, decreases with increasing temperature while the plateau modulus $G_{0}$ remains almost constant. The micellar length is proportional to the relaxation time, $\tau_{R}$ (see Eq. 1). Since $\tau_{R}$ decreases with temperature, it can be argued that micelles become shorter ${ }^{1,32)}$, and hence, the viscosity decreases.

On the other hand, in the case of $\mathrm{NaNO}_{3}$ system, the relaxation time increases on increasing temperature first slightly and then abruptly at $30^{\circ} \mathrm{C}$, which corresponds to the temperature at which the highest viscosity for the sample is attained. Further increase in temperature causes a decrease in the relaxation time. The plateau modulus also shows the same trend with an increase in temperature. Thus, it is inferred that first the micelle grows in one dimension and get entangled, causing an increase in vis- 


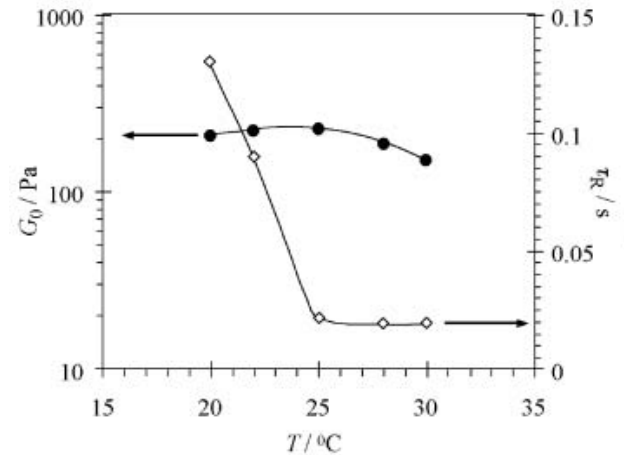

(a)

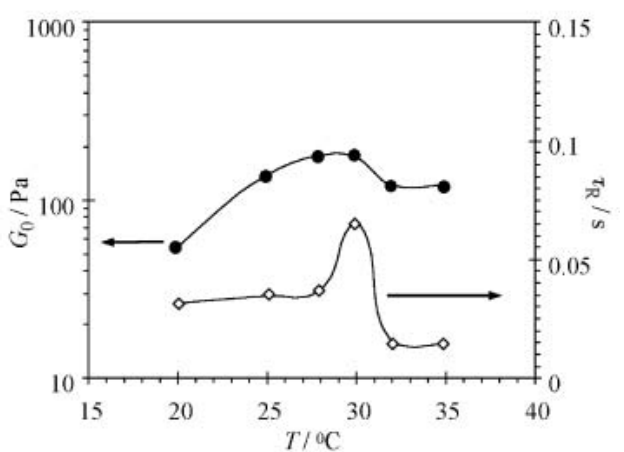

(b)

Fig. 9 Variation of Plateau Modulus, $G_{0}$ (circles), and Relaxation Time, $\tau_{\mathrm{R}}$ (diamonds) as a Function of Temperature $(T)$ for $10 \mathrm{wt} \% \mathrm{LAD}-$ Arg2/Water $+9.5 \mathrm{wt}_{0} \mathrm{C}_{14} \mathrm{EO}_{3}$ at Fixed Salt Concentration (1 M) for (a) $\mathrm{NaBr}$ and (b) $\mathrm{NaNO}_{3}$ Systems.

cosity, and then beyond $30^{\circ} \mathrm{C}$, the micelles are disrupted. Therefore, the viscosity of the sample decreases.

The studied systems are multicomponent, and therefore, several complex mechanisms could affect the thermoresponsive behavior. The temperature dependence of the Maxwellian relaxation time is usually described by an Arrhenius-type relationship:

$$
\tau_{R}=A \exp \left(\frac{E_{a}}{R T}\right)
$$

where $A$ is a pre-exponential factor, $R$ is the gas constant and $E_{a}$ is the total activation energy. The temperature dependence of zero-shear viscosity for a Maxwellian fluid could then be estimated by Eq. 4 , if $G_{0}$ is known as a function of temperature.

The decreasing part of the $\tau_{R}$ curve in Fig. 9a follows roughly Eq. 5, indicating a positive value of $E_{a}$ associated with micellar disruption. As a matter of fact, we found Arrhenius behavior in our previous report on worm-like micelles in salt-free $\mathrm{LAD}$-Lys $+\mathrm{C}_{14} \mathrm{EO}_{3}$ aqueous systems ${ }^{30}$. As the case of Fig. 9a, $G_{0}$ has been found to be rather temperature insensitive for cationic wormlike micelles ${ }^{38,39)}$. On the other hand, the maximum in the $\tau_{R}$ curve of Fig. 9b suggests the presence of two opposed contributions to $E_{a}$, namely, the activation energy could be expressed as $E_{a}=$ $E_{d}-E_{g}$, where $E_{d}$ and $E_{g}$ are activation energies for micellar disruption and growth, respectively. On this basis, for temperatures below the maximum, $E_{d}<E_{g}$, whereas for temperatures above the maximum, $E_{d}>E_{g}$. The difference in contributions of $E_{d}$ and $E_{g}$ depending on temperature could also serve as a qualitative explanation of the maximum in $\eta_{0}$ observed in Fig. 8.

The behavior of $G_{0}$ with temperature in $\mathrm{NaNO}_{3}$ systems also departs from previous reports on wormlike micelles. For dilute polymer solutions in a good solvent, it has been shown that ${ }^{40)}$.

$$
G_{0} \sim k_{B} T \xi^{-3} \sim k_{B} T \phi^{2.3}
$$

where $k_{B}$ is Boltzmann's constant, $\xi$ is the mesh size of the temporary network formed by the entangled polymer chains and $\phi$ is the polymer volume fraction. On the basis of Eq. 6, the trend for Fig. 9b could be only explained by a non-obvious dependence of $\xi$ on temperature.

The presence of nonionic surfactant, $\mathrm{C}_{14} \mathrm{EO}_{3}$ in the system can play a role in the increase of viscosity with temperature ${ }^{41)}$. In the case of nonionic surfactant system, on increasing the temperature, the dehydration of ethylene oxide chains induces a decrease in the interfacial curvature of aggregates. This in turn would lead to a sphere- rod transition in aggregate shape or promote one dimensional growth if the rod-like aggregates are already formed. The formation of end caps in the cylindrical aggregates becomes unfavorable with increasing temperature because of high free-energy cost of the formation of hemispherical ends, and consequently one-dimensional growth is favored. However, at high temperatures, due to a decrease in solubility, a migration of $\mathrm{C}_{14} \mathrm{EO}_{3}$ molecules towards the micellar core cannot be ruled out, which would contribute to the disruption of the long micelles ${ }^{42)}$.

\section{SUMMARY}

$\mathrm{N}$-dodecanoylglutamic acid neutralized by arginine in $1: 2$ mole ratio (LAD-Arg2) forms an aqueous micellar solution over a wide range of concentrations at $25^{\circ} \mathrm{C}$. Upon addition of lipophilic nonionic surfactant $\left(\mathrm{C}_{14} \mathrm{EO}_{3}\right)$ to the semi-dilute solution of the LAD-Arg2, the interfacial curvature decreases and LAD-Arg2 micelles grow, forming ultimately 
a transient network of viscoelastic wormlike micelles. Zero-shear viscosity $\left(\eta_{0}\right)$ increases with $\mathrm{C}_{14} \mathrm{EO}_{3}$ concentration continuously till a liquid crystal phase separates out. Dynamic oscillatory-shear rheological measurements have shown formation of wormlike micelles in the viscous regions, and the Maxwell model of single stress relaxation mode fits the data in the low frequency range.

Addition of salts enhances the micellar growth and shifts the $\eta_{0}$ curves towards lower $\mathrm{C}_{14} \mathrm{EO}_{3}$ concentration, which indicates that lower amount of $\mathrm{C}_{14} \mathrm{EO}_{3}$ is required for viscosity increase in the salt-added systems. In a particular salt system $\left(\mathrm{NaNO}_{3}\right)$, increasing salt concentration increases the maximum attainable zero-shear viscosity $\left(\eta_{0 \max }\right)$, but after a certain concentration, the $\eta_{0 \max }$ decreases. The increase in $\eta_{0 \max }$ can be attributed to the one dimensional micellar growth induced by screening effect of the salt. On the other hand, the decrease of $\eta_{0 \max }$ after the maximum could be attributed to micellar shortening or breaking, as indicated by the decrease in relaxation time, $\tau_{R}$. However, we cannot exclude the possibility of micellar branching.

Phase separation takes place at a certain temperature in salt-added systems studied; the phase separation temperature decreases in the order $\mathrm{I}^{-}>\mathrm{NO}_{3}{ }^{-}>\mathrm{Cl}^{-} \approx \mathrm{Br}^{-}$. The thermoresponsive rheological behavior was also found to be highly dependent on the type of anion, and anomalous trends, i.e. viscosity increase with temperature, were observed for all anions except $\mathrm{Br}^{-}$.

More detailed studies using other techniques (such as Cryo-TEM) are needed to get more insight into the origins of the observed rheological behavior.

\section{ACKNOWLEDGEMENT}

Authors are thankful to the Ajinomoto Co. Ltd. Japan for supplying the amino acid surfactant. Technical assistance of Dr. Lok K. Shrestha (Yokohama National University) is acknowledged. KA is thankful to Ministry of Education, Culture, Sports, Science and Technology, Grant-in-Aid for Young Scientists (B), No. 18780094 and partly supported by Core Research for Evolution Science and Technology (CREST) of JST Corporation.

\section{APPENDIX}

The effect of salt concentration on the steady-shear rheology, and hence, on $\eta_{0}$ for other salts $(\mathrm{NaCl}, \mathrm{NaBr}$ and $\mathrm{NaI}$ ) is also observed and is shown in Fig. A1. For the sake of comparison, data of the salt free system is also included in the figures.

The $\eta_{0}$ curves shift towards lower $\mathrm{C}_{14} \mathrm{EO}_{3}$ concentration with increasing salt concentration indicating that the salt

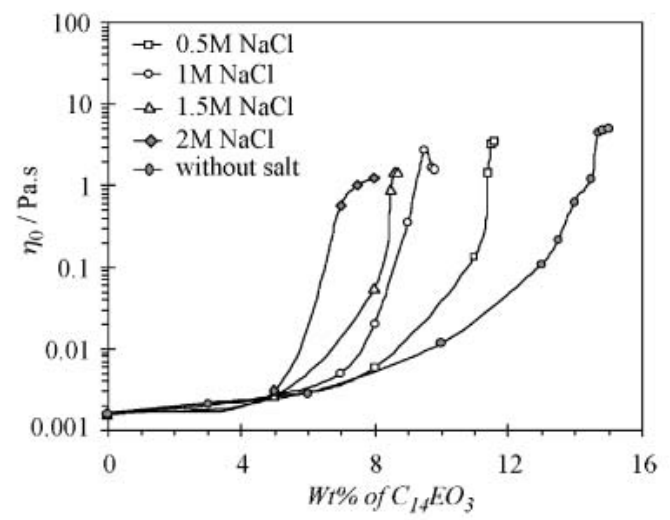

(a)

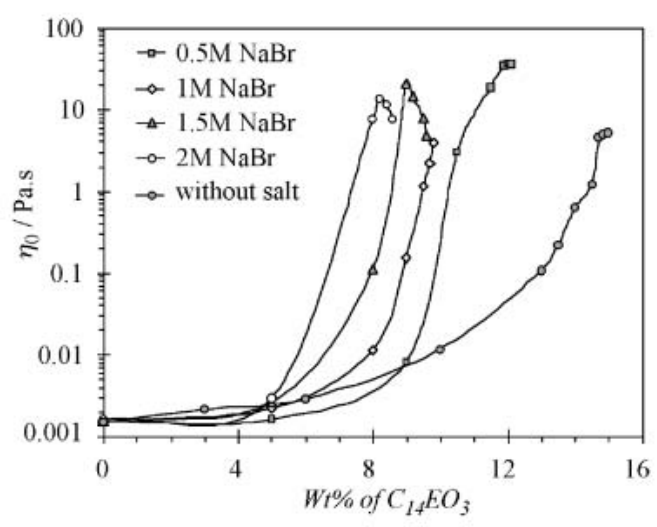

(b)

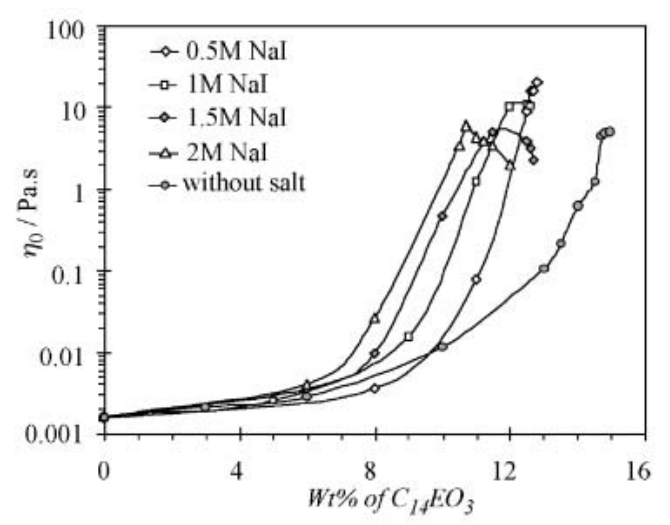

(c)

Fig. A1 Zero-shear Viscosity vs. $\mathrm{C}_{14} \mathrm{EO}_{3}$ Concentration at Different Concentrations of (a) $\mathrm{NaCl}$, (b) $\mathrm{NaBr}$ and (c)NaI.

The concentration of LAD-Arg2 is fixed at 10 $w t \%$. All the measurements were carried out at $25^{\circ} \mathrm{C}$. 


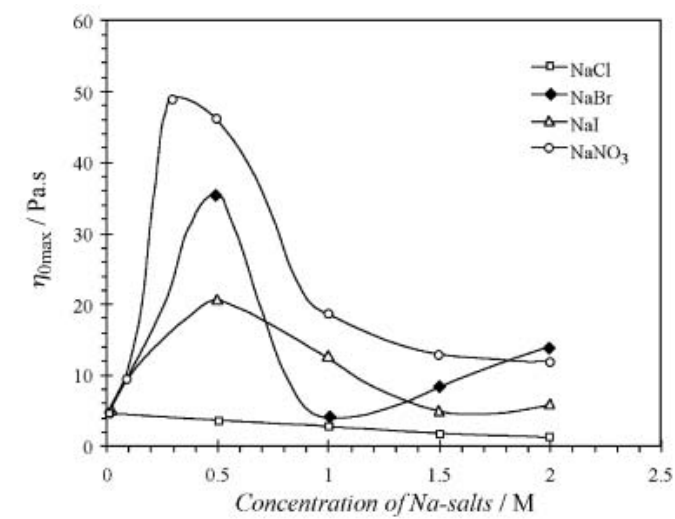

Fig. A2 The $\eta_{0 \max }$ as a Function of Na-salts Concentration. The concentration of LAD-Arg2 is fixed at 10 wt $\%$. All the measurements were carried out at $25^{\circ} \mathrm{C}$.

favors micellar growth. Curves shift to higher viscosities with increasing $\mathrm{C}_{14} \mathrm{EO}_{3}$ concentration. It is generally admitted that increasing the salt concentration amounts to an increase in the curvature energy of surfactant molecules in the end-cap region relative to the one in the cylindrical body of the micelle. This leads to an increase in micellar length.

The maximum attainable zero-shear viscosity, $\eta_{0 \max }$ as a function of salt concentration has been plotted for other $\mathrm{Na}$-salts too and presented in Fig. A2. As can be seen in the figure, the $\eta_{0 \max }$ first increases with $\mathrm{Na}$-salt concentrations, and then decreases. The viscosity increase can be attributed to one-dimensional micellar growth. On the other hand, the viscosity decrease is caused by micellar shortening or branching.

\section{References}

1. Cates, M.E.; Candau, S.J. Statics and dynamics of worm-like surfactant micelles. J. Phys. Condens. Matter 2, 6869-6892 (1990).

2. Rehage, H.; Hoffmann, H. Viscoelastic surfactant solutions: model systems for rheological research. Mol. Phys. 74, 933-973 (1991).

3. Hoffmann, H. Structure and flow in surfactant solutions in ACS Symp. (Herb, C.A.; Prudhomme, R. ed.). Ser. 578. ACS. Washington D.C pp.2-31 (1994).

4. Hoffmann, H.; Ulbricht, W. Transition of rodlike to globular micelles by the solubilization of additives. $J$. Colloid Interface. Sci. 129, 388-405 (1989).

5. Brackman, J.C.; Engberts, J. Polymer-induced breakdown of rodlike micelles. A striking transition of a nonNewtonian to a Newtonian fluid. J. Am. Chem. Soc. 112, 872-873 (1990).
6. Rehage, H.; Hoffmann, H. Rheological properties of viscoelastic surfactant systems. J. Phys. Chem. 92, 47124719 (1988).

7. Kern, F.; Lemarechal, P.; Candau, S.J.; Cates, M.E. Rheological properties of semidilute and concentrated aqueous solutions of cetyltrimethylammonium bromide in the presence of potassium bromide. Langmuir 8, 437-440 (1992).

8. Hassan, P.A.; Candau, S.J.; Kern, F.; Manohar, C. Rheology of wormlike micelles with varying hydrophobicity of the counterion. Langmuir 14, 6025-6029 (1998).

9. Shikata, T.; Shiokawa, M.; Imai, S. Viscoelastic behavior of surfactant threadlike micellar solutions: Effects of additives 4. J. Colloid Interface Sci. 259, 367-373 (2003).

10. Raghavan, S.R.; Fritz, G.; Kaler, E.W. Wormlike micelles formed by synergistic self-assembly in mixtures of anionic and cationic surfactants. Langmuir 18, 3797-3803 (2002).

11. Garg, G.; Hassan, P.A.; Aswal, V.K.; Kulshreshtha, S.K. Tuning the structure of SDS micelles by substituted anilinium ions. J. Phys. Chem. B 109, 1340-1346 (2005).

12. Yang, J. Viscoelastic wormlike micelles and their applications. Curr. Opin. Colloid Interface Sci. 7, 276-281 (2002).

13. Zakin, J.L.; Lu, B.; Bewersdorff, H.W. Surfactant drag reduction. Rev. Chem. Eng. 14, 253-320 (1998).

14. Lin, Z.Q.; Zakin, J.L.; Zheng, Y.; Davis, H.T.; Scriven, L.E.; Talmon, Y. Comparison of the effects of dimethyl and dichloro benzoate counterions on drag reduction, rheological behaviors, and microstructures of a cationic surfactant. J. Rheol. 45, 963-981 (2001).

15. Zhang, Y.; Qi, Y.Y:; Zakin, J.L. Headgroup effect on drag reduction and rheological properties of micellar solutions of quaternary ammonium surfactants. Rheol. Acta 45, 42-58 (2005).

16. Drappier, J.; Divoux, T.; Amarouchene, Y.; Bertrand, F.; Rodts, S.; Cadot, O.; Meunier, J.; Bonn, D. Turbulent drag reduction by surfactants. Europhys. Lett. 74, 362368 (2006).

17. Oda, R.; Narayanan, J.; Hassan, P.A.; Manohar, C.; Salkar, R.A.; Kern, F.; Candau, S.J. Effect of the lipophilicity of the counterion on the viscoelasticity of micellar solutions of cationic surfactants. Langmuir 14, 4364-4372 (1998).

18. Kim, W.J.; Yang, S.M.; Kim, M. Additive effects on the microstructure evolution in hexadecyltrimethylammonium bromide solution and its rheological properties. J. Colloid Interface Sci. 194, 108-119 (1997).

19. Mu, J.H.; Li, G.Z.; Jia, X.L.; Wang, H.X.; Zhang, G.Y. Rheological properties and microstructures of anionic micellar solutions in the presence of different inorganic salts. J. Phys. Chem. B 106, 11685-11693 (2002).

20. Shrestha, R.G.; Tobita, K.; Aramaki, K. Rheological 
behavior of viscoelastic wormlike micelles in mixed Ndodecyl glutamic acid/poly(oxyethylene) hexadecyl ether systems in presence of salts. Colloid \& Surfaces A 332, 103-111 (2009).

21. Acharya, D.P.; Sato, T.; Kaneko, M.; Singh, Y.; Kunieda, $\mathrm{H}$. Effect of added poly(oxyethylene)dodecyl ether on the phase and rheological behavior of wormlike micelles in aqueous SDS solutions. J Phys. Chem. B 110, 754-760 (2006).

22. Rodriguez, C.; Acharya, D.P.; Hattori, K.; Sakai, T.; Kunieda, H. Phase and rheological behavior of surfactant/novel alkanolamide/water systems. Langmuir 19, 8692-8698 (2003).

23. Acharya, D.P.; Hattori, K.; Sakai, T.; Kunieda, H. Phase and rheological behavior of salt-free alkyltrimethylammonium bromide/alkanoyl-N-methylethanolamide/ water systems. Langmuir 19, 9173-9178 (2003).

24. Kunieda, H.; Rodriguez, C.; Tanaka, Y.; Kabir, Md.H.; Ishitobi, M. Effects of added nonionic surfactant and inorganic salt on the rheology of sugar surfactant and CTAB aqueous solutions. Colloids \& Surface B 38, 127-130 (2004).

25. Engelskirkchen, S.; Acharya, D.P.; Garcia-Roman, M.; Kunieda, $\mathrm{H}$. Effect of $\mathrm{C}_{12} \mathrm{EO}_{\mathrm{n}}$ mixed surfactant systems on the formation of viscoelastic worm-like micellar solutions in sucrose alkanoate- and CTAB-water systems. Colloid \& Surfaces A 279, 113-120 (2006).

26. Sikata, T.; Hirata, H.; Kotaka, T. Micelle formation of detergent molecules in aqueous media. 3. Viscoelastic properties of aqueous cetyltrimethylammonium bromide-salicylic acid solutions. Langmuir 5, 398-405 (1989).

27. Imae, T. Light scattering of spinnable, viscoelastic solutions of hexadecyltrimethylammonium salicylate. J. Phys. Chem. 94, 5953-5959 (1990).

28. Schubert, B.A.; Kaler, E.W.; Wanger, N.J. The microstructure and rheology of mixed cationic/anionic wormlike micelles. Langmuir 19, 4079-4089 (2003).

29. Shrestha, R.G.; Shrestha, L.K.; Aramaki, K. Formation of wormlike micelle in a mixed amino-acid based anionic surfactant and cationic surfactant systems. J. Colloid Interface Sci. 311, 276-284 (2007).

30. Shrestha, R.G.; Shrestha, L.K.; Aramaki, K. Wormlike micelles in mixed amino acid-based anionic/nonionic surfactant systems. J. Colloid Interface Sci. 322, 596-
604 (2008).

31. Kaneko, D.; Olsson, U.; Sakamoto, K. Self-assembly in some N-lauroyl-l-glutamate/water systems. Langmuir 18, 4699-4703 (2002).

32. Granek, R.; Cates, M.E. Stress relaxation in living polymers: Results from a poisson renewal model. J. Chem. Phys. 96, 4758-4767 (1992).

33. Larson, R.G. The structure and rheology of complex fluids; Oxford University Press: New York, (1999).

34. Marin, G. Rheological Measurement, 2nd edn. (Collyer, A.A.; Clegg, D.W. Eds.). Chapman and Hall. London (1998).

35. Doi, M.; Edwards, S.F. The theory of polymer dynamics. Oxford University Press. Clarendon, U.K. (1986).

36. Freitag, R.; Garret-Flaudy, F. Salt effects on the thermoprecipitation of poly-(N-isopropylacrylamide) oligomers from aqueous solution. Langmuir 18, 34343440 (2002).

37. Weckstrom, K.; Papageorgiou, A.C. Lower consolute boundaries of the nonionic surfactant C8E5 in aqueous alkali halide solutions: An approach to reproduce the effects of alkali halides on the cloud-point temperature. J. Colloid Interface Sci. 310, 151-162 (2007).

38. Kern, F.; Zana, R.; Candau, S.J. Rheological properties of semidilute and concentrated aqueous solutions of cetyltrimethylammonium chloride in the presence of sodium salicylate and sodium chloride. Langmuir 7, 1344-1351 (1991).

39. Tung, S.H.; Huang, Y.-E.; Raghavan, S.R. Contrasting effects of temperature on the rheology of normal and reverse wormlike micelles. Langmuir 23, 372-376 (2007).

40. Adam, M.; Delsanti, M. Viscosity and longest relaxation time of semi-dilute polymer solutions. I. Good solvent. J. Phys. (Paris) 44, 1185-1193 (1983).

41. Acharya, D.P.; Sharma, S.C.; Rodriguez-Abreu, C.; Aramaki, K. Viscoelastic micellar solutions in nonionic Fluorinated Surfactant Systems. J. Phys. Chem. B 110, 20224-20234 (2006).

42. Rodriguez, C.; Aramaki, K.; Tanaka, Y.; Lopez-Quintela, M.A.; Ishitobi, M.; Kunieda, H. Wormlike micelles and microemulsions in aqueous mixtures of sucrose esters and nonionic cosurfactants. J. Colloid Interface Sci. 291, 560-569 (2005). 